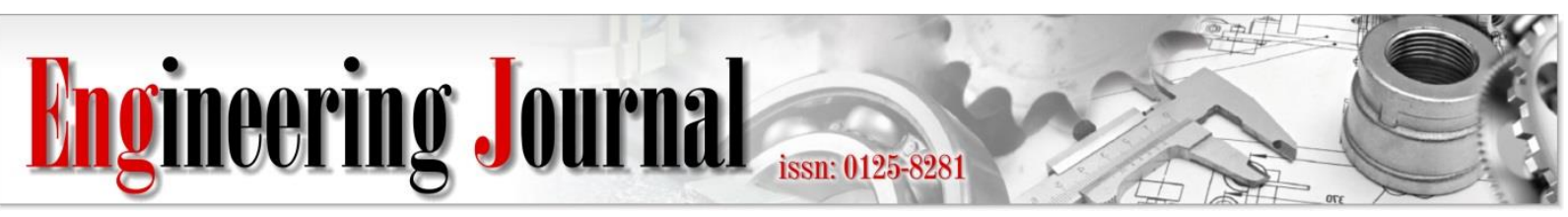

Article

\title{
Development of Novel Wind Turbines Hybridized between Permanent Magnet Disks and Additional Motor/Generator for Extending Operating Range and Enhancing Conversion Efficiency
}

\author{
Rungsiman Kulpetjira ${ }^{1, *}$, Jeeraphun Kulpetjira ${ }^{1}$, and Suwit Kiravittaya ${ }^{2}$ \\ 1 Phitsanulok Pittayakom School, 1 Moo 8, Ta Tong Sub-District, Muang District, Phitsanulok Province, \\ Thailand \\ 2 Department of Electrical and Computer Engineering, Faculty of Engineering, Naresuan University, \\ Ta-Pho Sub-District, Muang District, Phitsanulok Province, Thailand \\ *E-mail: severip101@hotmail.com (Corresponding author)
}

\begin{abstract}
We implement and evaluate a novel wind turbine setup, which consists of a conventional wind turbine and a pair of coupled disks. The disks are coupled by permanent magnets (PMs) and the distance between the coupling disk pair can be varied. The force between disks has been simulated as a function of disk distance and magnetic field strength. The strongest repulsive force is produced when the PM disks are in-line but when the PM disks are interdigitated, the coupled force is changed to attractive force and the coupling is maximized. Our testing experiment shows that when the wind speed is too low, the coupled disks can transfer angular speed to the turbine in order to maintain the turbine movement. With these added parts, we can avoid the rotational barrier from static friction when the wind speed is increased again. Without the coupled disks, the turbine will completely stop when the wind speed is too low. The static turbine needs stronger wind to start the movement again. Extension of the utilization of the turbine to the highwind-speed situation has also been proposed. This work enhances the usage of wind turbine for producing renewable energy in low-wind-speed regions.
\end{abstract}

Keywords: Wind energy, wind turbine, permanent magnet coupling.

ENGINEERING JOURNAL Volume 25 Issue 1

Received 1 August 2020

Accepted 25 November 2020

Published 31 January 2021

Online at https:/ / engj.org/

DOI:10.4186/ej.2021.25.1.23 


\section{Introduction}

Nowadays, utilization of renewable power and energy becomes ubiquitous [1-3]. Wind energy is a kind of renewable energy that has been integrated into the electrical generation and distribution systems in many countries [2-4]. Globally, the generated wind power in 2018 reaches 564 GW (from the total generated renewable energy of $2351 \mathrm{GW}$ ) [5]. Wind turbine farms have been continuously installed around the world. However, in many countries including Thailand this kind of energy resource is not practical because of several limitations $[3,6]$. The randomness nature of wind speed and direction produces unstable energy flow in the generating system based on pure wind [7]. Energy storage might be used to relieve this problem, but it does increase the system cost and complexity. Many researchers have developed novel hybrid systems for overcoming this problem. For example, Sun et al. have demonstrated that the energy storage in term of compressed air is feasible to integrate with a wind turbine system [8]. Nowadays, many kinds of integrative power systems and circuits have also been developed and demonstrated for improving the energy conversion of the overall systems [9-11].

In any turbine system, coupling is an important part to transmit mechanical power from the turbine to electrical generator. Simple coupling system consists of only shaft, which directly connect the turbine and generator on a single axis. For more complicated systems, gearbox and mechanical brake system might be installed in order to control the turbine. In many designs, permanent magnet (PM) has been utilized in the coupling system because it has advantages that there is no mechanical contact involved [12-17]. Force can be transferred via induced magnetic field. Hallbach design has been widely adapted for this purpose [12-15]. Eddy current is usually generated in such systems. In a novel design of Potgieter et al., PM coupling has been used for coupling both turbine and stator [18]. In addition, this PM coupling can also be used for braking function. To our knowledge, PM coupling technology has not been specifically tested in any conventional wind turbine systems.

Here, we propose a novel concept of hybrid wind turbine. It has advantages for both light-wind and strongwind situation as compared with a typical turbine system. Qualitative comparison is presented in Fig. 1. Typically, a wind turbine can operate in a wind speed range between cut-in and cut-out speeds. The turbine can generate amount of electric power depending on the turbine speed [2-4]. The cut-in wind speed is needed because the turbine at rest must overcome the static friction in the system while the turbine cannot operate above the cutout speed due to the safety reason. For the latter case, the maximum turbine speed must be set based on the mechanical design of the system. With the hybrid wind turbine, we can extend the operating range. The cut-in speed can be shifted to the lower value by avoiding the static friction and the second generator can be applied to increase the generated power and reduce the turbine speed. Both functionalities can be integrated to a conventional wind turbine by using coupling disks connected with motor/turbine.

In this work, we investigate the PM coupling disk by first simulating the magnetic field to qualitative describe the coupling behavior. A prototype of hybrid wind turbine is implemented in this work and the experimental testing of the system in light wind situation is done. A promising result is obtained. Algorithm for operating this hybrid wind turbine for both low-wind-speed and highwind-speed situations is proposed. The paper is divided into 7 sections. They are 1. Introduction, 2. Simulation of Magnetic Coupling Force, 3. Prototype Development, 4. Experimental Setup, 5. Results in Low-wind-speed Scenario, 6. Proposed Diagram for Automatic Operation and 7. Conclusion.

\section{Simulation of Magnetic Coupling Force}

In order to evaluate the magnetic coupling, a simulation of magnetic field distribution from the PM disks is performed. Figure 2(a) is a schematic drawing of the simulated PM disks installed on solid circular plates. The PM disks are axially aligned. The distance $d$ and the mismatch angle $\theta$ between them are varied in this work. For the simulation we use a finite-element-method (FEM) based software package to simulate the magnetic field and calculate the force [19]. Details of the structural
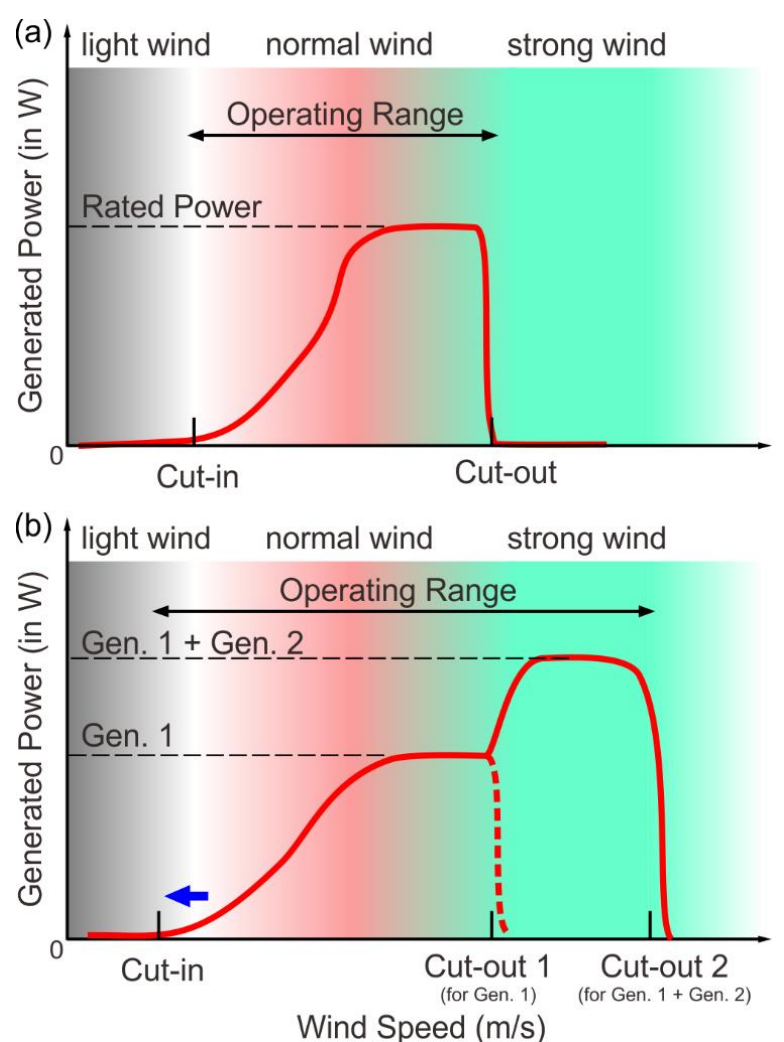

Fig. 1. Schematics of generated power vs. wind speed in (a) a conventional wind turbine and (b) the proposed hybrid wind turbine. 
(a)

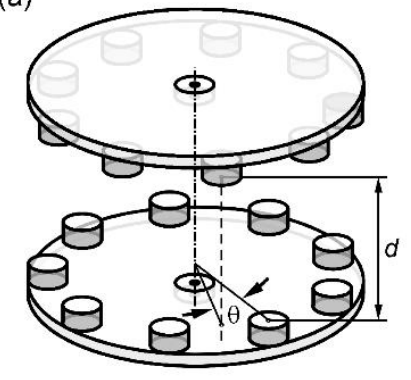

(b)

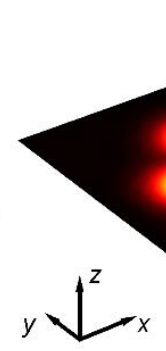

(c)

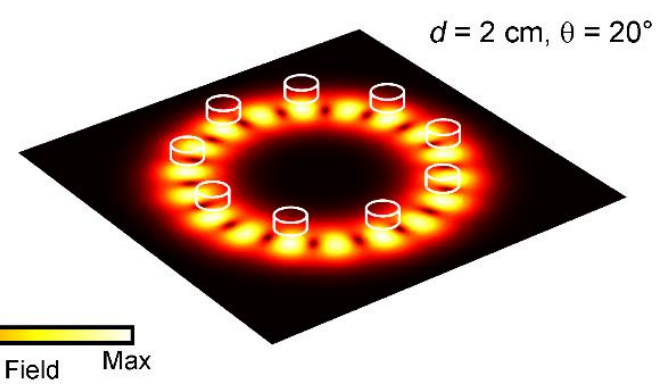

Fig. 2. (a) Schematic of the simulated coupling disks. Magnetic field distribution on the middle plane when between the disks when the magnets are (b) aligned and (c) interdigitated.

parameters are as follows. The solid circular plates have the diameter of $17 \mathrm{~cm}$. Nine cylindrical PMs (diameter of $19 \mathrm{~mm}$ and height of $8.9 \mathrm{~mm}$ ) are installed on the inner edge of each circular plate. The angular distance between PMs is $40^{\circ}$. Therefore, $\theta$ can be varied from $0^{\circ}$ (perfectly aligned) and $20^{\circ}$. In the latter state, the PMs on the coupling disks are interdigitated. Magnetic strength in term of equivalent magnetic flux density is fixed at $1 \mathrm{~T}$ for each PM. North pole of all PMs on the upper PM disk are faced down to the north pole of the PMs on the lower PM disk.

Figure 2(b) shows the results from the magnetic field simulation on the middle plane between the PM disks for $d=2 \mathrm{~cm}$ and $\theta=0^{\circ}$. It shows that magnetic field is distributed into a ring shape for each PM pair because the strong magnetic repulsion between the same poles (north) of each pair is induced in this configuration. This configuration thus exhibits the unfavorable coupling of the PM disks.

Figure 2(c) shows the simulation results for $d=2 \mathrm{~cm}$ and $\theta=20^{\circ}$. The magnetic field distribution largely changes as compared with the former case (Fig. 2(b)). The magnetic field from each PM are interdigitated uniformly along azimuthal direction. The result implies the preference of the coupling between these coupling disks. The PM in upper and lower disks are furthest apart at this configuration and therefore the north pole of each PM can interact with the opposite pole on another side of the nearby PMs.

To quantify the induced magnetic force between PM disks, the vertical and horizontal forces are calculated as a function of both $d$ and $\theta$. They are calculated from the obtained magnetic field vectors [20, 21], i.e.,

$$
\mathbf{F}=\frac{1}{2} \int(\mathbf{H}(\mathbf{n} \cdot \mathbf{B})+\mathbf{B}(\mathbf{n} \cdot \mathbf{H})-\mathbf{n}(\mathbf{B} \cdot \mathbf{H})) \mathrm{d} A=0
$$

where $\mathbf{F}$ is the derived force vector $(\mathrm{N}), \mathbf{H}$ is the magnetic field strength $(\mathrm{A} / \mathrm{m}), \mathbf{B}$ is the magnetic flux density ( $\mathrm{T}), \mathbf{n}$ is the unit normal vector, and $A$ is the integrated area which is the middle plane.
Figure 3(a) shows the extracted vertical force between coupling disks at different angle for the distance $d=1,2$, and $3 \mathrm{~cm}$. The repulsive force (positive value) is generally observed because of the same PM polarity. The maximum repulsive force is observed when the PM disks are aligned and it monotonically increases when the distance decreases. When the angle $\theta$ is varied, the change of the sign from repulsive force to attractive force is observed. For the distance $d=1 \mathrm{~cm}$, the attractive force occurs at $\theta$ between $12^{\circ}$ and $28^{\circ}$. This indicates the coupling ability of the disks.
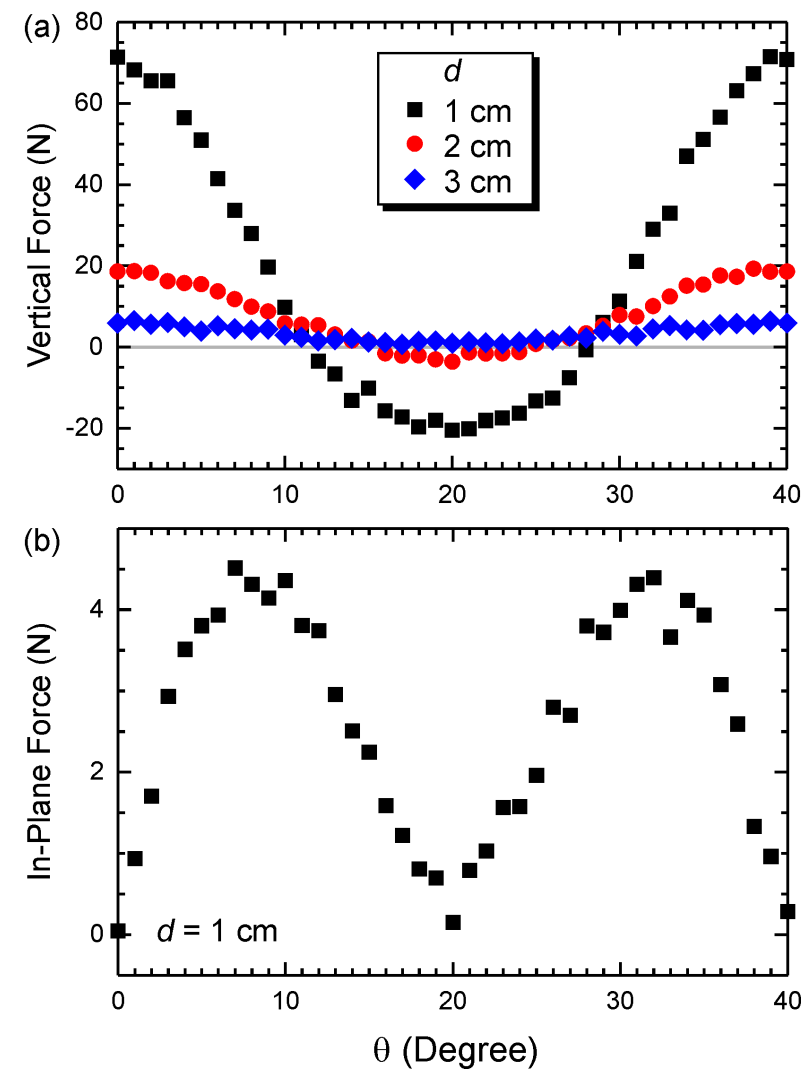

Fig. 3. (a) Calculated vertical pushing force between the disks when the distance $d$ is 1,2 , and $3 \mathrm{~cm}$. (b) Calculated in-plane pushing force between the disk when $d=1 \mathrm{~cm}$. Zero in-plane force is observed at $\theta=0^{\circ}$ and $20^{\circ}$. 


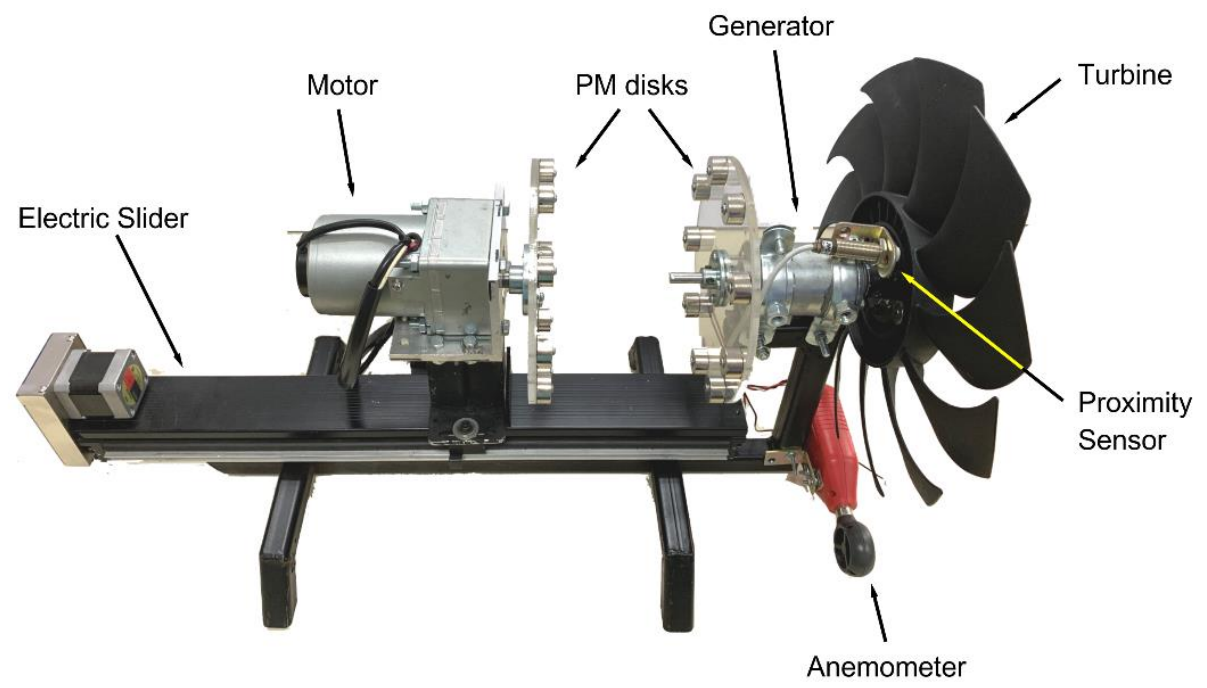

Fig. 4. Photo of prototype of the developed hybrid wind turbine. It consists of a pair of coupling PM disks, motor, electric slider, generator, turbine, proximity sensor and anemometer.

Figure 3(b) shows the in-plane pushing force between the disk at the distance $d=1 \mathrm{~cm}$. When the disks are either perfectly aligned $\left(\theta=0^{\circ}\right)$ or perfectly interdigitated $\left(\theta=20^{\circ}\right)$, the in-plane force disappears because of the symmetrical cancellation of all in-plane attractive/ repulsive forces. Maximum in-plane force occurs at the angles of $7^{\circ}-10^{\circ}$ and $30^{\circ}-33^{\circ}$. The asymmetric force profile originates from the geometries of both PMs and the coupling disk. At these angles, the disk prefers to rotated away in order to reduce this force. In combination with the vertical force, the disk will prefer to couple in the interdigitated states (at $\theta=20^{\circ}$ ).

\section{Prototype Development}

The prototype developed and further tested in this work consists of a generator connected with a turbine and a coupling disk. Another coupling disk is connected with a DC motor installed on a linear electric slider. Wind speed and turbine speed are measured with an anemometer (UNI-T UT363) and a proximity sensor, respectively. A photo of the prototype is shown in Fig. 4.

Coupling PM disks consist of 9 pieces of donut shape neodymium iron boron grade N42. They are installed on the inner edge of $1 \mathrm{~cm}$ thick acrylic disk. The first PM disk connects to the generator and the turbine with the same shaft. Another PM magnet disk connects to the speed-controlled motor (Oriental DSC Series 6W speed $18-320 \mathrm{rpm}$ ). The linear electric slider consists of THK Caged ball LM series guide actuator and Vexta oriental stepping motor at the base. This slider is for moving the motor connected with PM disk to couple or uncouple with another PM disk.

\section{Experimental Setup}

Schematic diagram of the setup for testing experiment is shown in Fig. 5. Controlled and constantspeed wind is generated from a commercial fan. The measuring part consists of a 4-channel digital oscilloscope (Keysight MSOX2014A) and a computer. The wind speed is measured by the anemometer, which is installed below the turbine. The signal is sent to the oscilloscope. The turbine speed is measured by the proximity sensor. The wind power is converted to the voltage signal at the generator. The signals from both proximity sensor and the generator are sent to the same oscilloscope. The motor and electric slider are separately controlled. The control box is developed to allow a manual control of both DC motor speed and the distance between two coupling disks. All signals are recorded and analyzed offline by using a computer with the MATLAB program [22]. 


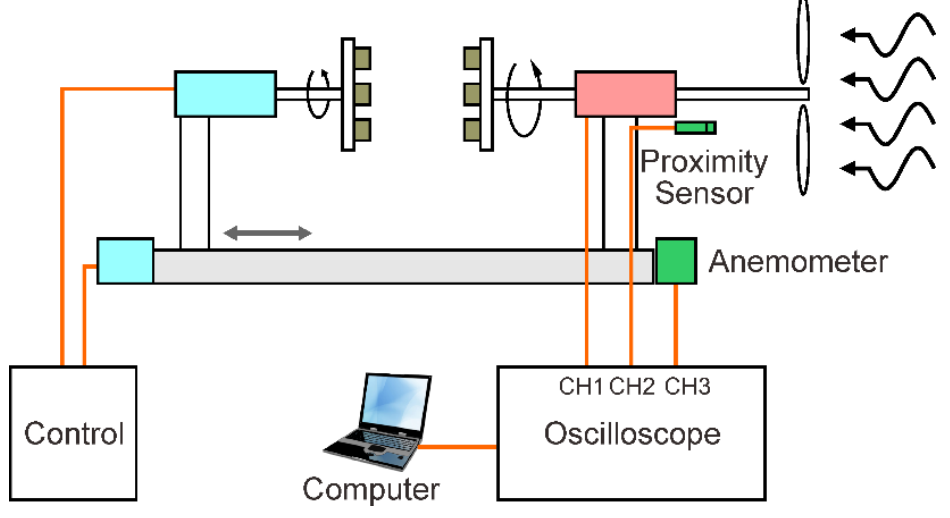

Fig. 5. Schematic diagram of the setup for testing experiment.

\section{Results in Low Wind Speed Scenario}

Typical voltage signals from the generator, proximity sensor and anemometer are shown in Fig. 6 for three wind speeds. In Fig. 6(a), the average voltages (18.34, 24.74 , and $28.33 \mathrm{~V}$ ) can relate to the turbine speeds (469, 638 , and $728 \mathrm{rpm}$ ). The higher voltage is obtained when the input wind is stronger. However, the voltage signals are not constants. The observed oscillation is due to the internal pole inside the generator. This oscillation frequency is proportional to the turbine speed as well. To accurately measure the turbine speed, signal from the proximity sensor is measured as shown in Fig. 6(b). The width of the pulse $d t_{B}$ and the period is inversely proportional to the turbine speed. From this signal, one can measure the turbine speed by the measuring the pulse width or the signal period.

In Fig. 6(c), the voltage signals from the anemometer are recorded. Both pulse width $d t_{C}$ and the signal period are inversely proportional to the wind speed. The wind speeds are 3.6, 4.0, and $4.4 \mathrm{~m} / \mathrm{s}$. A linear relation between the wind speed and the turbine speed is assumed in our test.
The experiment comparison between normal wind turbine and wind turbine with rotating disk coupling is presented in Fig. 7. In the tested situation, strong-wind to no-wind and light-wind scenario is set. Figure 7(a) shows normal wind turbine without rotating disk coupling. The result shows that when the turbine faces with strong wind the turbine speed increases and become stable in the first 30 second. Then, by switching off the wind, the turbine speed decrease to zero (in the next 18 second). Later, when the light wind presented, the turbine is still not rotated because the wind speed is still below the cut-in speed of the turbine.

For the same turbine with the rotating disk coupling, the result is shown in Fig. 7(b). If the rotating disk is coupled to the turbine when the light wind is presented, the turbine can initiate its rotation and the wind can maintain it. One can gain the wind power in this scenario if the light wind scenario occurs in a long-time scale. The energy is needed for initially rotating the turbine. However, we believe that one can gain more energy when the light wind does continuously flow.
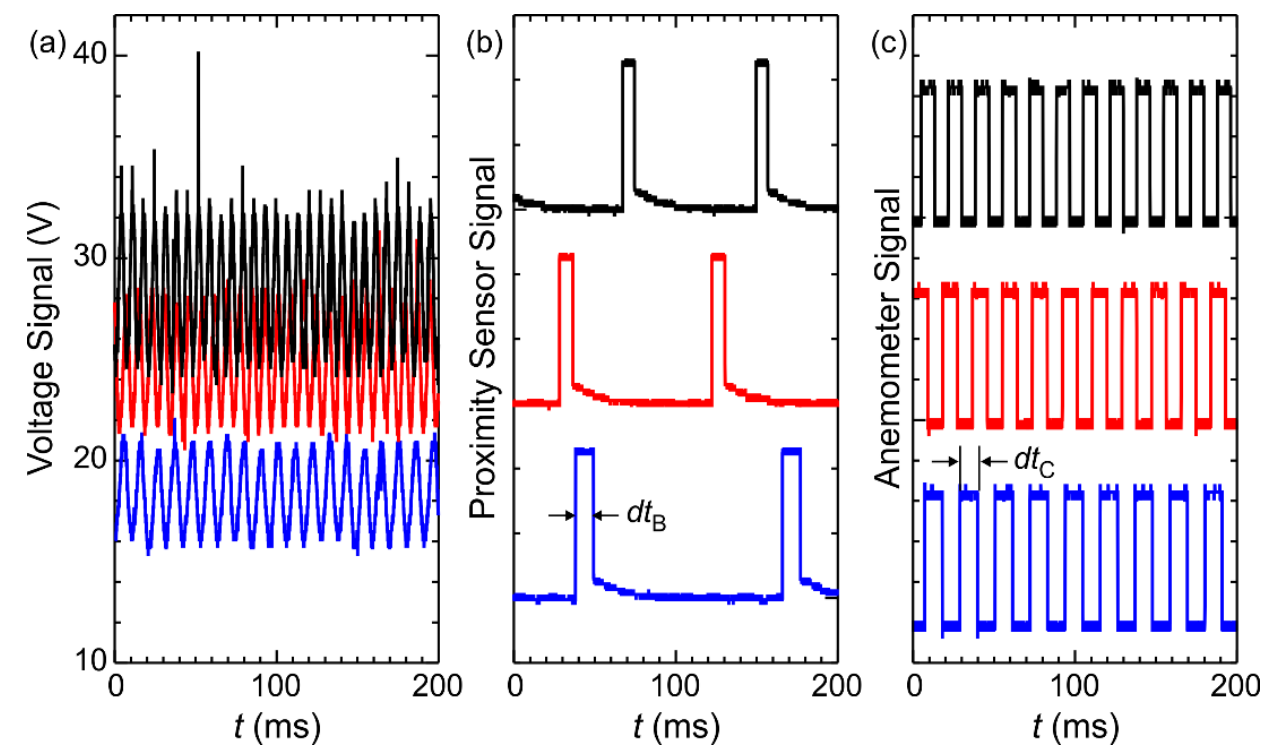

Fig. 6. The recorded signals at three different wind speeds: (a) voltage signal at the generator terminal, (b) proximity sensor signal and (c) anemometer signal. 

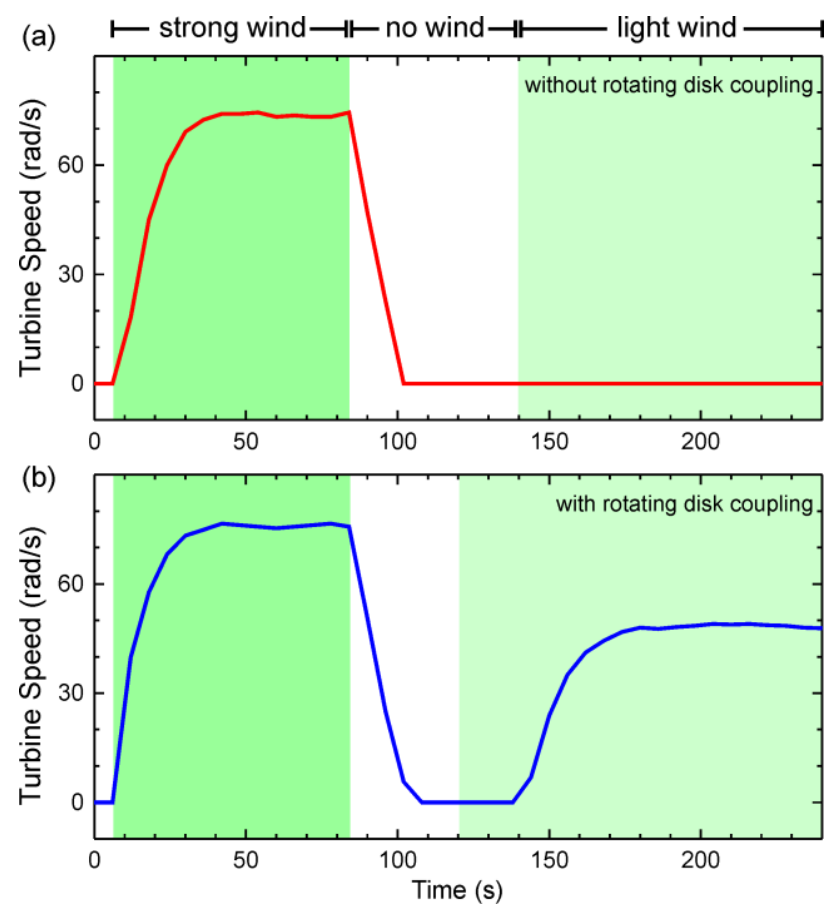

Fig. 7. The recorded turbine speeds in case that the rotating PM disk is (a) unused and (b) coupled when the wind speed is low.

\section{Proposed Diagram for Automatic Operation}

According to the result shown in the previous section and the concept described in the introduction (Fig. 1), an automatic operation of the hybrid wind turbine can be proposed. The proposed diagram for automatic operation is shown in Fig. 8. The operational system starts by getting the signal from proximity sensor then convert signal from digital pulse to turbine speed. If turbine speed is lower that constant value $\omega_{\min }$, coupling process will do. The procedure is drive speed control motor at turbine speed. Next, the electric slider is driven to the coupling position that PM disks are closed together so the magnetic field can transfer force together. Next, the signal from anemometer is read if the wind speed is more than a preset constant value $V_{w \text {,min }}$ or minimum wind speed that turbine can rotate. The electric slider will move to uncoupling position that PM disk far together so the magnetic field cannot transfer force together then stop the speed control motor. If the wind speed is lower than constant value $V_{m, m i n}$, wind speed is checked again and redo the whole process.

In case of high wind, the system is operated by measuring signal from proximity sensor and then convert it to wind turbine speed. If the turbine speed is faster than the maximum angular speed, the linear slider will be moved the generator with PM disk close to another PM disk. The coupling disks can couple each other and reduce the turbine speed. If the wind speed is slower than maximum angular speed, the linear slider will separate generator from wind turbine for uncoupling.

If the turbine speed is slower than minimum angular speed, it means the low wind condition occurred. The speed-controlled motor will rotate at wind turbine speed then the linear slider will move the motor to couple it with turbine. The motor will remain the turbine speed at minimum angular speed. If the wind speed is slower than minimum angular speed. The linear slider will separate motor from wind turbine for uncoupling and stop the motor. 


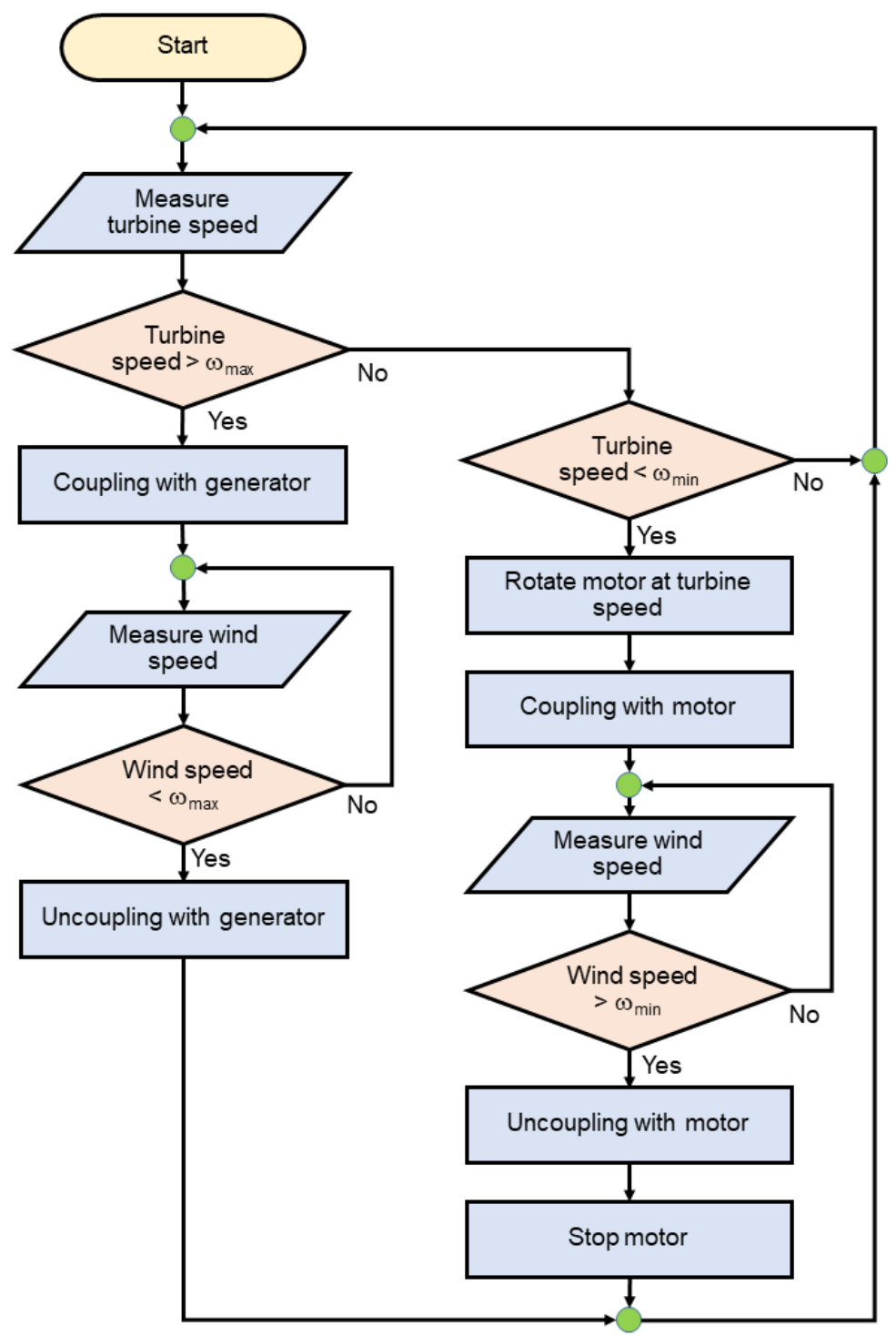

Fig. 8. The flowchart of the proposed control algorithm.

\section{Conclusions}

This work presents a proposed concept for extending the operation range of a wind turbine. First, a simulation study of the PM coupling disks is performed. The prototyped hybrid wind turbine is realized and tested with low-wind-speed scenario. It has been shown that the developed hardware can induce the turbine rotation in light wind scenario. Diagram for operating the hybrid wind turbine in both light and strong wind scenarios is proposed. This work enhances the development of renewable energy technology.

\section{Acknowledgement}

This research work is financially supported by Junior Science Talent Project (JSTP) Scholarship, National Science Technology Development Agency (NSTDA), Thailand (Contract no. SCA-CO-2559-1621). We thank the SMC Thailand Company and Faculty of Engineering Naresuan University for their equipment used in this research, Prof. Phadungsak Ratanadecho and Assistant Prof. Dr. Boonchoat Paosawatyanyong are acknowledged for their fruitful discussion. 


\section{References}

[1] G. D. Price, "Photovoltaic systems Volume I," in Renewable Power and Energy Volume 1. Momentum Press, LLC, 2018.

[2] G. D. Price, "Wind and thermal systems," in Renewable Power and Energy Volume 2. Momentum Press, LLC, 2018.

[3] S. Heier, "Onshore and offshore conversion systems," in Grid Integration of Wind Energy, 3rd ed. John Wiley \& Sons, Ltd, 2014.

[4] A. John and P. Brian, "Small-scale wind power," in Environmental Engineering Collection. Momentum Press, LLC, 2014.

[5] A. Whiteman, J. Esparrago, S. Rueda, S. Elsayed and I. Arkhipova, "Renewable capacity statistics 2019," International Renewable Energy Agency, 2019.

[6] S. Chingulpitak and S. Wongwises, "Critical review of the current status of wind energy in Thailand," Renewable and Sustainable Energy Reviews, vol. 31, pp. 312-318, 2014.

[7] F. Porte-Agel, M. Bastankhah, and S. Shamsoddin, "Wind-turbine and wind-farm flows: A review," Boundary-Layer Meteorology, vol. 174, pp. 1-59, 2020.

[8] H. Sun, X. Luo, and J. Wang, "Feasibility study of a hybrid wind turbine system-Integration with compressed air energy storage," Applied Energy, vol. 137, pp. 617-628, 2015.

[9] Z. Chen, J. M. Guerrero, and F. Blaabjerg, "A review of the state of the art of power electronics for wind turbines," IEEE Transactions on Power Electronics, vol. 24, no. 8, pp. 1859-1875, 2009.

[10] F. Blaabjerg and K. Ma, "Wind energy systems," Proceedings of the IEEE, vol. 105, no. 11, pp. 21162131, 2017.

[11] A. Zahedi, "Sustainable power supply using solar energy and wind power combined with energy storage," Energy Procedia, vol. 52, pp. 642-650, 2014.

[12] K. Halbach, "Design of permanent multipole magnets with oriented rare earth cobalt materials,"
Nuclear Instruments and Methods, vol. 169, pp. 1-10, 1980.

[13] J.-M. Kim, J.-Y. Choi, M.-M. Koo, H.-J. Shin, and S.-H. Lee, "Characteristic analysis of tubular-type permanent-magnet linear magnetic coupling based on analytical magnetic field calculations," IEEE Transactions on Applied Superconductivity, vol. 26, no. 4, p. 0604605, 2016.

[14] H.-J. Shin, J.-Y. Choi, S.-M. Jang, and K.-Y. Lim, "Design and analysis of axial permanent magnet coupling based on 3D FEM," IEEE Transactions on Magnetics, vol. 49, no. 7, pp. 3985 - 3988, 2013.

[15] Z. Di, W. Dazhi, S. Tongyu, and L. Zhen, "Design optimization of permanent magnet couplings," in Proceedings of 27th Chinese Control and Decision Conference (CCDC), 2015.

[16] R. Romain, L. Valerie, and L. Guy, "Analytical design of permanent magnet radial couplings," IEEE Transactions on Magnetics, vol. 46, no. 11, pp. $3860-3865,2010$.

[17] J.-Y. Choi, H.-J. Shin, S.-M. Jang, and S.-H. Lee, "Torque analysis and measurements of cylindrical air- gap synchronous permanent magnet coupling based on analytical magnetic field calculations," IEEE Transactions on Magnetics, vol. 49, no. 4, pp. 3921 - 3924, 2013.

[18] J. H. J. Potgieter and M. J. Kamper, "Optimum design and comparison of slip permanent-magnet couplings with wind energy as case study application," IEEE Transactions on Industry Applications, vol. 50, no. 5, pp. 3223 - 3234, 2014.

[19] COMSOL. Available: https://www.comsol.com/

[20] F. Mach and P. Karban, "Dynamic behavior of electromagnetic brake system consisting of permanent magnets," Electrical Review, vol. 87, no. 5, pp. 100-103, 2011.

[21] J. A. Stratton, Electromagnetic Theory. Hoboken, New Jersey: John Wiley \& Sons, Inc., 2007.

[22] MATLAB. https://www.mathwork.com/ 


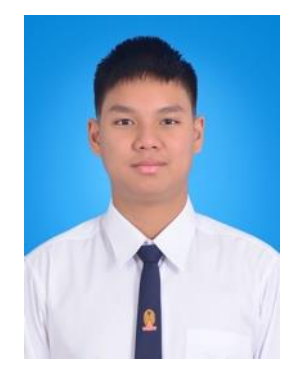

Rungsiman Kulpetjira was born in Bangkok, Thailand in 2001. He graduated from Phitsanulok Pittayakrom School, Phitsanulok, Thailand in 2018. He has currently received Junior Science Talent Project scholarship from National Science and Technology Development Agency since 2014.

From 2014 to 2018, he interested in pneumatic systems and technologies. He has invented an automatic air-conditioner cleaner.

Rungsiman Kulpetjira has received The-Third Place Grand Award from Intel International Science and Engineering Fair in 2019, and Gold medal from Seoul International Invention Fair in 2017 and 2018.

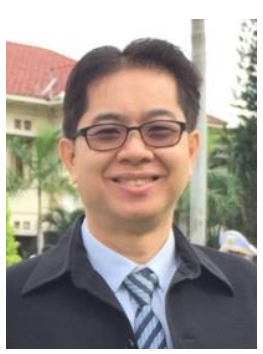

Jeeraphun Kulpetjira was born in Phichit, Thailand in 1971 . He received the B.S. degree in Chemical Science from Rajamangala University of Technology Krungthep, Bangkok, Thailand in 1994.

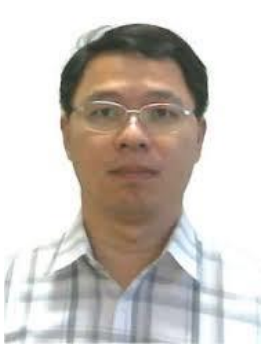

Suwit Kiravittaya was born in Bangkok, Thailand, in 1976. He received the bachelor and doctoral degrees in Electrical Engineering from Chulalongkorn University, Bangkok, in 1998 and 2003, respectively. Since 2018, he has been an Associate Professor with the Department of Electrical and Computer Enginerring, Faculty of Engineering, Naresuan University, Phitsanulok, Thailand. He is the author of six books and more than 130 publications in both national and international journals. His research interests are 1) Applied optics and electronics for various systems, 2) Opto-electronics: solar cells, photodetectors, LEDs and lasers, 3) Molecular beam epitaxy and optical properties of semiconductor nanostructures, and 4) Quantum information technology: computation, communication and coding. 